\title{
LA CLASE DE FUNCIÓN 'COMPLEMENTO CIRCUNSTANCIAL' DE MODO EN ESPAÑOL
}

\author{
María Luisa Masiá Canuto \\ (Universidad Jaume I, Castellón)
}

\begin{abstract}
RESUMEN
The starting point of this research is to be found in essays on linguistic functions carried out by E. Alarcos, G. Rojo and S. Gutiérrez. It was in the field of linguistic functionalism that the question of semiotic nature of linguistic functions was explicity stated: if utterance syntactic relations are meaningful, such meanings must be associated to significants which perform the task of transmitting them. Alarcos, Rojo and Gutiérrez began their work with subject function. Agustín Vera continued such study by also defining direct and indirect object function.

Although we have studied adverbial function in its wholeness. this article is intended only to state our work on manner adverbials. Significant and meaning of manner class is a metalinguistic sign which we shall have to study.
\end{abstract}

Este trabajo es una propuesta de definición de la clase de función 'complemento circunstancial' de modo desde una perspectiva semiótica-funcional. Nuestra intención es caracterizar a esta clase de función como un esquema sígnico, al igual que lo realizaron ALARCOS (1977), ROJO (1979), GUTIÉRREZ (1983) y A. VERA (1988-89)- (1990) con la categoría de función sujeto.

A. VERA (1990:35) ha sintetizado perfectamente la cuestión con estas palabras:

Si las relaciones sintácticas que operan en el seno de la oración son significativas, parece lógico suponer que tales significados están asociados a unos significantes que se encargan de transmitirlos, por lo que tales relaciones deben poder ser referidas a unos esquemas de signos de los que deberán determinarse todos sus componentes. 
Veamos, pues, algunas de las propuestas de caracterización de la función sujeto como esquema sígnico realizadas por ALARCOS (1977 a: 13-15), ROJO (1979: 137 y ss.) y GUTIÉRREZ (1983:50-52):

\begin{abstract}
ALARCOS
sustancia expresión

forma expresión

forma contenido

sustancia contenido
\end{abstract}

ROJO

sustancia expresión

forma expresión

forma contenido

sustancia contenido

\section{GUTIÉRREZ ORDÓÑEZ}

Función abstracta

sustancia expresión

forma expresión

forma contenido

sustancia contenido

Función concreta

forma expresión

forma contenido concordancia

sujeto

agente

concordancia

sujeto

agente

agente
SN concomitante en $n^{\circ}$ y pna. con $S V$

SN concordante en $n^{\circ}$ y pna. con SV

sujeto

/Juan, llave, el viento, los ancianos.../ sujeto

Juan

agente

A. VERA (1988-89 y 1990) matizó algunas de estas afirmaciones. En primer lugar, dice VERA, la asociación entre sujeto y agente en la formulación de ROJO puede resultar problemática, ya que no todos los sujetos funcionan como agentes (podrían poseer el valor de experimentador (1)). Si respetamos esta asociación, el principio de indisociabilidad de expresión y contenido no se podría mantener.

\section{(1) El animal recibió una patada.}

En segundo lugar, las tres propuestas poseen cierta deficiencia explicativa al incorporar el término que desean definir, 'sujeto', en la propia definición. A. VERA piensa que esto podría evitarse si consideramos las funciones como signos metalingüísticos. El significante de este signo sería la categoría de función 'sujeto', su significado lo constituiría a su vez otro signo. 


\section{SIGNO METALINGÜÍSTICO}

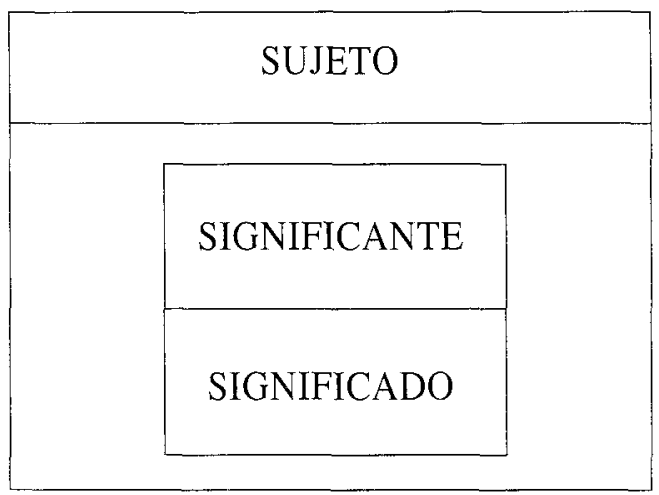

Enmarcada así la categoría de función 'sujeto', VERA propuso la siguiente caracterización de este signo. En primer lugar, para establecer el significado lingüístico VERA estudia el papel funcional de esta relación sujeto para con el todo oracional del que forma parte. Esta es la formulación de la concepción oracional de A. VERA (1990: 41 y ss.):

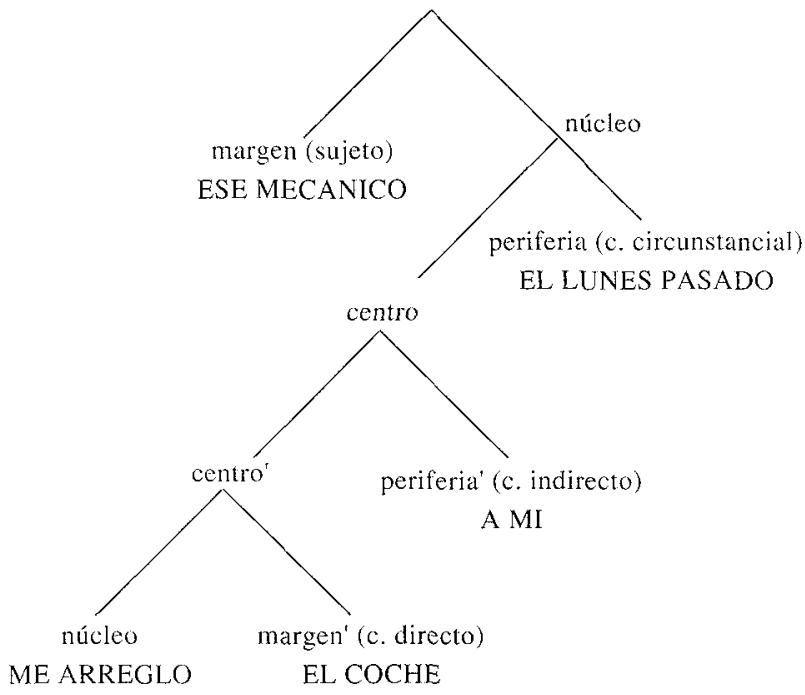

La estructura interna de la oración obedece, como indicábamos, a una jerarquización compleja en la que, en primer lugar, es posible diferenciar entre un núcleo y un margen como tipos de relaciones que caracterizan a predicado y sujeto, respectivamente. Para la existencia de la unidad oración, en efecto, es imprescindible la existencia del primero, pero no la del segundo. 
El significado del significado metalingüístico será, por lo tanto, MARGEN.

Para la formulación del significante, VERA tuvo en consideración la concepción que R. TRUJILLO (1976:161) presenta sobre éste. Para R. TRUJILLO, un significante no lo constituyen exclusivamente los aspectos fónicos, sino todos aquellos rasgos que puedan llegar a ser señales de la existencia de un significado. La 'concordancia en número, persona y caso nominativo' será para VERA el rasgo definidor idóneo del significante. La categoría de función 'sujeto' quedaría, pues, de la siguiente forma:

\begin{tabular}{|c|}
\hline Categoría sujeto \\
\hline $\begin{array}{c}\text { Concord. } \mathrm{n}^{\circ}, \text { pna., } \\
\text { caso nominativo }\end{array}$ \\
\hline Margen \\
\hline
\end{tabular}

Vera definió de forma similar las categorías de función 'complemento directo' e 'indirecto'. El significado del significado metalinguiístico del complemento directo es, como habíamos visto ya, MARGEN'. El significado linguístico del comple-mento indirecto es PERIFERIA'.

En cuanto a los significantes correspondientes, VERA los formulará tras analizar el comportamiento de los pronombres personales que pueden desempeñar estas funciones. Por un lado, estudia la relación de presuposición sintáctica existente entre las formas pronominales átonas y las formas pronominales tónicas: las segundas suponen siempre la presencia de las primeras. Resultan, por consiguiente, agramaticales aquellos enunciados oracionales en los que no esté presente la forma átona correspondiente. Si lo que está ausente es la forma tónica, no hay agramaticalidad. Los ejemplos siguientes pueden ilustrar esta afirmación:

(2) Pedro lo insultó áél

(2i) *Pedro insultó a él

(2ii) Pedro lo insultó.

(3) El barbero le afeitó a él la barba

(3i) *El barbero afeitó a él la barba

(3ii) El barbero le afeitó la barba 
Por otro lado, VERA defiende la existencia de una conjugación objetivapersonal, siguiendo las propuestas de K. HEGER (1974) y A. LLORENTE-J. MONDÉJAR (1974): es posible hallar en el verbo español monemas en relación morfemática con él, de contenido personal, numeral, etc. Por tanto, del mismo modo que hablamos de la existencia de una 'concordancia en número, persona y caso nominativo entre el verbo y el sujeto, podemos hacerlo de una concordancia entre los sintagmas verbales y los pronombres personales en función de complemento directo e indirecto. En el complemento directo, la relación de concordancia entre los pronombres átonos constituyentes del núcleo predicativo (lo $(\mathrm{s})$, $\underline{\mathrm{la}}(\mathrm{s})$ ) y sus correspondientes tónicos es de género, número, persona y caso acusativo. La concordancia es similar en el complemento indirecto, si exceptuamos el caso y el género. El caso es el dativo, y no hay marca de género presente en los pronombres átonos correspondientes (le $(s))$.

(4) El niño lo lee a él con interés (el libro)

(5) Antonio la arrojó a ella a la piscina (a Victoria)

(6) Les prestaron su ayuda a ellos

(7) Les prestaron su ayuda a ellas

Veamos, pues, cómo quedarán definidos:

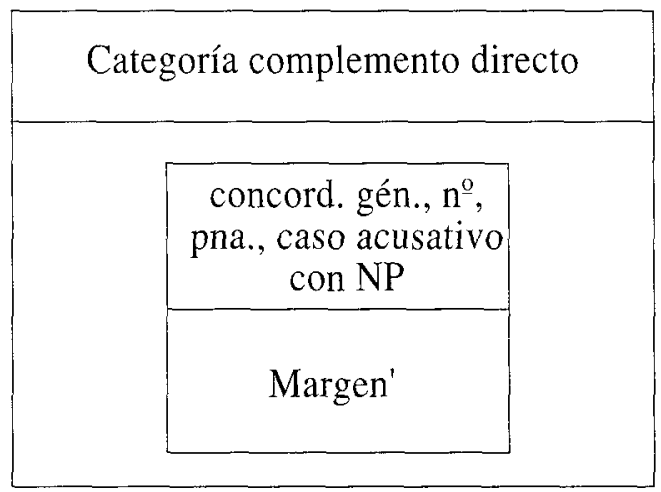




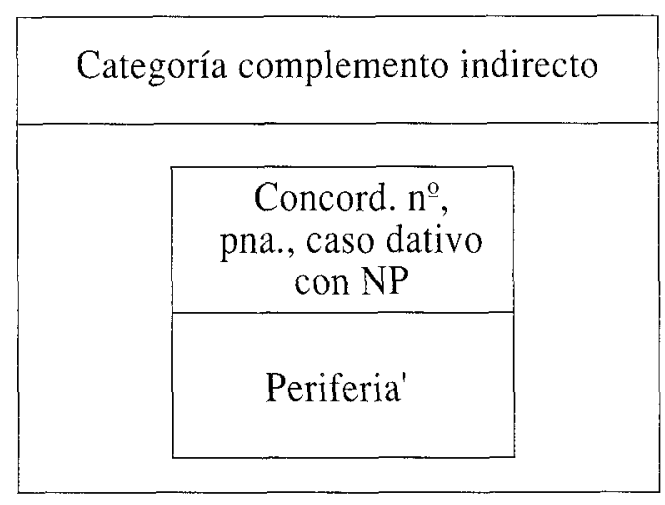

En esta línea iniciamos nuestro estudio sobre la categoría de función 'complemento circunstancial' y sus correspondientes clases de función' . Para la determinación del significado del plano del contenido del signo metalinguístico categoría de función 'complemento circunstancial', la mayoría de caracterizaciones existentes eran de escasa utilidad. La consideración de estos complementos como transmisores de circunstancias no es una propiedad «categorial», en cuanto que no está definiendo el papel del complemento circunstancial respecto del todo en el que se integra. Resultaría más adecuada la propuesta que defiende la marginalidad como característica de esta función. Sin embargo, también son eliminables sintagmas con funciones distintas:

(8) Andrés pintaba acuarelas.

(8i) Andrés pintaba

(9) Le contó a ella una historia

\section{(9i) Contó una historia}

Sin duda, el papel de PERIFERIA que hemos asignado anteriormente al complemento circunstancial cumple los requisitos necesarios para poder caracterizar el significado lingüístico de esta categoría de función. La naturaleza gramatical de este significado se corresponde con la naturaleza sintáctica de las funciones. El rasgo de PERIFERIA no supone otra cosa que estar hablando de

Vid. A. VERA LUJÁN-M" L. MASIÁ CANUTO, «La categoría de función 'complemento circunstancial'en español» Voz y letra, II, 1, 1991, pp. 51-75. 
una función sintáctica oracional constituyente del predicado, pero con una vinculación a éste menor que la de las restantes funciones predicativas como lo demuestra enunciados como (10), en el que el circunstancial desde París quedaría fuera del dominio de lo hice, el cual si incluye al objeto directo e indirecto:

(10) Mi hermana escribió una carta a nuestros primos desde Málaga, y yo lo hice desde París.

Para poder determinar el rasgo que nos permitiría caracterizar el significante de ese significado, revisamos las propuestas realizadas hasta la fecha, y en muchas de ellas observamos una carencia de intencionalidad sistematizadora. Así J. ALCINA- J. M. BLECUA (1975: 889), S. GILI GAYA (1948: 62, 183 y 186), J. ROCA PONS (1980: 289-290), R.A.E. (1974: 376) o C. HERNÁNDEZ ALONSO (1984: 88-89) se habían limitado a enumerar los tipos sintagmáticos que pueden desempeñar esta función: adverbiales, preposicionales y nominales. Frente a ellos, algunos lingüistas como L. TESNIËRE (1976: 103), E. ALARCOS LLORACH (1973: 221 y 1986: 4), H. MARTÍNEZ GARCÍA (1986: 40), ROJO (1985: 183) O E. RAMÓN TRIVES (1990:566) presentaban una voluntad de abstracción, y seleccionaban el tipo adverbial como invariante representativa. Podríamos, por tanto, haber identificado el significante con la condición adverbial de los sintagmas que puede funcionar como complementos circunstanciales. No obstante, para que hubiese sido posible mantener esta concepción, el significante de los complementos directo e indirecto habría de haber sido caracterizado mediante sintagmas nominales y preposicionales. Si esto no se realizaba así, no podría existir una base común entre los significantes que permitiera establecer las relaciones opositivas que otorgan valor a estas unidades.

Finalmente, consideramos que no era ésta la única y más adecuada manera de definir los significantes de los complementos directo, indirecto y circunstancial. Hasta ahora, el rasgo de concordancia había caracterizado todos y cada uno de los significantes definidos. Por ello nos preguntamos si existía alguna relación similar entre los sintagmas pronominales-personales en función de complemento circunstancial, que nos permitiera caracterizar esta función del mismo modo. Detengámonos en estos ejemplos:

(11) Su maestro los compara contigo

(12) Dirigió terribles insultos contra él

(13) Le hizo eso ante mí

(14) Puso la mano enél 


\section{(14i) *Le puso la mano en él}

Como podemos observar, el núcleo del predicado no puede presentar ninguna forma átona del mismo modo que ocurría en las funciones complemento directo e indirecto. Si esta forma átona existiera supondría una reiteración en el núcleo del predicado de los mismos contenidos de los pronombres personales que funcionan como complemento circunstancial. La agramaticalidad de los enunciados (11i)-(14i) es debida, pues, a la no existencia de esta forma átona. Un rasgo identificador del complemento circunstancial podía ser la ausencia de esta concordancia. El complemento circunstancial quedaba así definido:

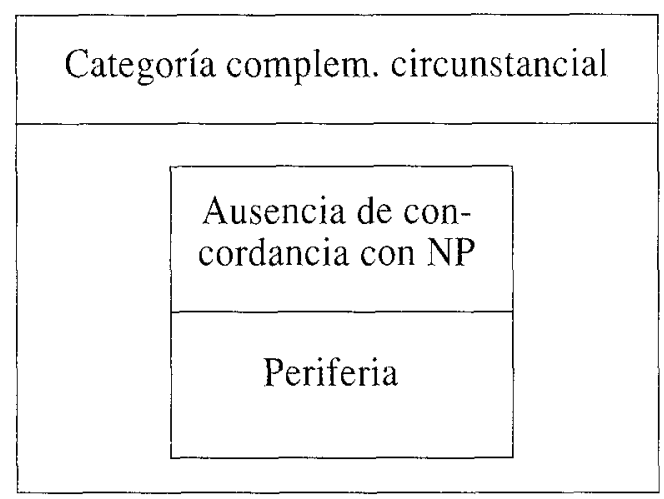

Una vez establecida la definición de la categoría de función 'complemento circunstancial', es hora ya de estudiar las clases de función 'complemento circunstancial'. Nuestro concepto de clase de función y categoría de función se basa en la diferenciación de ROJO entre relaciones parte-todo y relaciones parteparte. ROJO (1983:51) piensa que las funciones sintácticas oracionales deben ser consideradas desde una doble perspectiva: relaciones parte-todo y relaciones parte-parte. Siguiendo este planteamiento y la propuesta de A. VERA (1988-89) distinguimos, pues, en este estudio entre categorías de función (funciones consideradas desde la perspectiva de las relaciones parte-todo) y clases de función 
(funciones consideradas como relaciones parte-parte). Por tanto, nuestra labor ahora no será otra que la de analizar las valencias sintagmáticas de estas clases de función, es decir, las relaciones de compatibilidad e incompatibilidad de esta función.

Consideramos que una forma adecuada de definir el significado lingüístico de la clase de función de modo es MODO. En cuanto al significante del complemento circunstancial de modo pensamos que presenta los siguientes rasgos:

El contenido modal puede ser expresado mediante sintagmas preposicionales, adverbios, locuciones adverbiales, construcciones de gerundio y participio, y las proposiciones subordinadas adverbiales introducidas por las conjunciones como, según (que), según (y) como, según y conforme, etc.

(15) « (...) observaron cómo los «marines» sacaban las armas de la zona tan pronto como estuvo controlada.» (El Mundo, 8-1-93, p. 17)

(16) Según sea vuestro comportamiento este fin de semana, iremos o no al cine el próximo lunes.

(17) Según y cómo esté el mar, podré o no partir el martes

(18) Según y conforme a lo dispuesto en el Código Civil, la compraventa se perfecciona por el mero consentimiento entre las partes contratantes.

A) Si el complemento circunstancial es generado mediante sintagmas preposicionales, éstos se construirán encabezados por las siguientes preposiciones: a, con, en, sin.

A: Puede introducir un sintagma preposicional de lugar, tiempo o modo. Si expresa lugar, el sintagma nominal introducido por esta preposición puede ser substituido por un adverbio de lugar (19/19i). En cambio, si su valor es temporal, todo el sintagma preposicional podrá ser substituido por un adverbio de tiempo (20). Si no puede ser realizada ninguna de estas substituciones, su valor será de modo (21).

(19) Mi casa siempre ha estado a la derecha de algún supermercado / Mi casa siempre ha estado allí

(19i) «En medios palestinos prevalece la opinión de que si Gorakan regresa a Nueva York con las manos vacías (...)» (El Mundo, 8-1-93, p. 15)/En medios palestinos prevalece la opinión de que si Gorakan regresa allí con las manos vacías.

(20) José Luis pasó por aquí a última hora / José Luis pasó por aquí muy tarde. 
(21) «(...) al irrumpir a punta de pistola en el Congreso de los Diputados» (El Mundo, 8-1-93, p. 9)

EN: Puede introducir un sintagma preposicional de lugar, tiempo o modo. $\mathrm{Si}$ indica lugar todo el sintagma preposicional podrá ser sustituido por un adverbio de lugar (22). Si es de tiempo la sustitución la llevará a cabo un adverbio de tiempo (23). En el resto de casos será de modo (24).

(22) «Los vientos huracanados extienden la marea negra en Escocia» (El Mundo, 8-1-93, p. 1) / Los vientos huracanados extienden la marea negra alli.

(23) En este momento nos lo estamos pasando muy bien / Ahora nos lo estamos pasando muy bien.

(24) No aguanto más en cuclillas. ¿Nos vamos?

CON: Puede introducir un sintagma preposicional de instrumento, compañía o modo. Para expresar el contenido de instrumento, esta preposición ha de emplearse exclusivamente con verbos de acción (25). Si expresa compañía, el sintagma nominal que se articula tras esta preposición podrá ser reemplazado por un pronombre personal tónico (26). Si indica modo, podrá complementar no sólo a verbos de acción (27), sino también a otros verbos, como los verbos de experimentación ${ }^{2}$ (28). Con los núcleos de predicado de acción, los complementos podrán expresar circunstancias relativas a la responsabilidad, control y volición.

(25) «Juan Piña se dio varios cortes con el mismo machete de submarinismo con el que había asesinado a Ana María (...) » (El Mundo, 8-1-93, p. 23)

(26) «El Rey intimó con Fernando Almansa (...)» (El Mundo, 8-1-93, p. 10)/El Rey intimó con él.

(27) No disimules, el golpe me lo has dado con toda intención

(28) Tu madre ve con gusto nuestro baile

2 Según CHAFE (1976:162-63), las experimentaciones son situaciones dinámicas que implicann una determinada actitud mental. 
SIN: Puede introducir un sintagma preposicional de instrumento, compañía o modo. Si el complemento es instrumental, esta preposición ha de emplearse con verbos de acción (29). Si expresa compañía, el sintagma nominal que se articula tras esta preposición podrá ser reemplazado por un pronombre personal tónico (30). Si indica modo, podrá complementar a verbos de experimentación (32), de estado (33), y de acción (31). Con este último tipo de núcleo de predicado, los complementos podrán expresar circunstancias relativas a la responsabilidad, control y volición.

(29) La tortilla debe comerse sin el cuchillo

(30) «Su mujer lo había acompañado por todos los caminos durante tanto tiempo que no podía recordar la vida sin ella.» (Allende, I., De amor y de sombra, Barcelona, Plaza \& Janés, 1991, p. 31)

(31) « Siempre he procurado estar quieto y servir sin ostentación en puestos oscuros (...)»(El Mundo, 8-1-93, p. 9).

(32) Mi hermana aprende con facilidad cualquier lengua

(33) Antonio fue golpeado sin tregua por aquel grupo de niños

B) Adverbios y locuciones adverbiales:

(34) «La respuesta del guardia civil golpista fue colgar el teléfono bruscamente» (El Mundo, 8-1-92, p. 9).

(35) Ahora me encuentro perfectamente en tu casa

C) Construcciones de gerundio y participio :

(36) «Así lo dicen en Zarzuela, condensando en esas dos palabras, «la casa», una llaneza doméstica, (...) » (El Mundo, 8-1-93, p. 10)

(37) « Unidades fuertemente armadas de «marines» norteamericanos apoyadas por carros de combate y helicópteros lanzaron un ataque (...) » (idem)

(38) « Tras caer dos metros por la trampilla, colgado de una soga la cual había sido tratada con gases especiales.» (El Mundo, 8-1-93, p. 23).

Consideramos necesario diferenciar dentro de la clase de modo, tres subclases: 
1) La primera estaría formada por aquellos complementos de modo, generalmente opcionales, que acompañan a verbos de acción y estado. Estos complementos contienen, tal y como señala A. VERA (1990: 124-134), circunstancias relativas a la responsabilidad, control y volición. El contenido modal lo presentan, por supuesto, pero a diferencia de la subclase segunda, en esta subclase primera, hemos encontrado estos matices de responsabilidad, control y volición. (39)-(40)

2) La segunda estaría formada por los complementos de modo, generalmente también opcionales, que acompañan, como apunta A. VERA (1990: 124-134), a verbos de experimentación (41).

3) En tercer lugar, tendríamos la subclase de los complementos que acompañan, no de manera opcional, a verbos de estado y a verbos llamados por $\mathbf{I}$. BOSQUE 'pseudocopulativos' (42)-(43). En esta subclase, hemos encontrado un matiz de estimación o valoración en el contenido modal.

(39) El niño aplastó su nariz contra el cristal intencionadamente/ El niño aplastó su nariz contra el cristal

(40) La puerta fue abierta voluntariamente por el portero / La puerta fue abierta por el portero

(41) Mi hermana ve con agrado una película todas las semanas /Mi hermana ve una pelicula todas las semanas

(42a) Esta comida sabe a rayos / * Esta comida sabe. (42b) Nunca te encuentras bien $\gg / * N u n c a$ te encuentras

(43) El vestido te queda muy bien / *El vestido te queda

I. BOSQUE (1991: 139-142) ha estudiado en profundidad estas construcciones con verbos 'pseudocopulativos' (subclase 3 ):

(...) los Ilamados 'pseudocopulativos', que designan, como se ha señalado con frecuencia, el resultado o el mantenimiento de alguna acción o algún proceso (...). Podemos pues suponer que los $<<$ resultados $>>$ forman parte de la estructura argumental de tales predicados estativos. De esos resultados se predican precisamente los adjetivos y los adverbios. (...).Lo que estos adverbios valorativos ponen de manifiesto es que lá lengua los admite como categorías sintácticas apropiadas para predicarse de los resultados de las acciones o de los procesos, en ausencia de las acciones o de los procesos mismos.

Para poder caracterizar de esta forma el significado y el significante lingüístico hemos revisado los estudios existentes hasta el momento. En ellos hemos encontrado dos carencias fundamentales. En primer lugar, la mayoría de ellos, a 
la hora de realizar sus propuestas, no se fundamentan en criterios sintácticos sino en criterios semánticos. En segundo lugar, es frecuente encontrar en ellos una falta de adecuación explicativa.

Consideramos que nuestra propuesta consigue superar estas carencias. Por un lado, nuestra caracterización de las clase modal se realiza dentro de una perspectiva de gran interés explicativo. Por otro lado, hemos intentado no caer en taxonomismos basados exclusivamente en criterios semánticos. Revisemos a modo de ejemplo uno de los intentos más exhaustivo de clasificación de estos complementos modales: este estudio no es otro que el aparecido en QUIRK ET AL. (1972: 426:470). GREENBAUM (1964) y QUIRK et alii (1972) establecieron una diferenciación entre adjuncts, disjuncts y conjuncts. Los conjuncts no son objeto de este estudio debido a que no consideramos que los conectores discursivos sean complementos circunstanciales. La diferencia planteada por estos lingüistas entre adjuncts y disjuncts es muy similar a la de complementos del enunciado o de la enunciación Recordemos que BALLY $(1965,47)$, representante de la escuela ginebrina, estudió un posible comportamiento alternativo para los adverbios: algunos adverbios no modifican el dictum sino al verbo performativo implícito en toda oración (modus). Trabajos posteriores han desarrollado este comportamiento. Merecen ser destacados los estudios de GREENBAUM (1969), SCHREIBER (1971), ALLERTON, D.J.-GRUTTENDEN, A. (1974), LAPESA (1978), BARRENECHEA (1979), EGEA (1979), RAMÓN TRIVES (1982), KOKTOVA (1986), KOVACCI (1980), FUENTES (1987).

Según KOVACCI (1980-1981:519-21), el dictum es lo común al significado de las oraciones (44)-(46), las cuales son diferentes en su modus:

(44) Va a llover

(45) ¿Va a llover?

(46) Que llueva

El modus se realiza, pues, a través del tono, el modo, índices léxicos (por ejemplo que, ojalá) etc, e indica tanto la presencia del hablante como su actitud con respecto a lo dicho.

La misma forma adverbial que puede modificar al modus, puede modificar al dictum:

(47) Todo se resolvió felizmente. (modificador del dictum)

(48) Todo se resolvió, felizmente. (modificador del modus)

Las paráfrasis de estas oraciones nos pueden ayudar a captar su diferente naturaleza: 
(47i) Fue felizmente (o de manera feliz) como se resolvió todo.

(48i) Es una felicidad que todo se resolviera

La diferenciación entre complementos modales de enunciado y de enunciación, en realidad, no tiene cabida en nuestra investigación. Tanto como modificador de predicado como de oración, el comportamiento sintáctico de estos complementos es similar. No importa si la circunstancia alude al $<<$ decir $>>0$ a $<<$ lo dicho $>>$. Si es al $<<$ decir $>>$, el complemento acompaña a un verbo de acción (declaración, interrogación, mandato, etc). Si por el contrario es a $<<$ lo dicho $>>$, podrá acompañar o a un verbo de acción o de cualquier otra naturaleza.

\section{(49) Usted no trabaja, porque el suelo está sucio}

(49i) Digo que no trabaja porque el suelo está sucio.

Esto mismo es aplicable en el resto de complementos circunstanciales.

Pero va a ser en el establecimiento de las diferentes subclases entre los complementos adjuncts, disjuncts y conjuncts, cuando son tenidos en cuenta exclusivamente criterios semánticos. Siguiendo estos criterios, se distinguen seis subclases dentro del grupo de los complementos adjuncts : viewpoint, focusing, intensifier, process, subject, y formulaic. Pensamos que si tenemos en cuenta criterios sintácticos, nuestras tres subclases serían suficientes. Veámoslo:

1) Viewpoint

(50) «Moralmente, políticamente, y económicamente, es urgente que el gobierno actúe con mayor eficacia en la ayuda al desarrollo de los paises. » (p. 429)

Este complemento quedaría englobada en nuestra subclase 1, ya que en realidad estamos ante un complemento de modo que modifica a un verbo de acción (afirmo):

(50i) «Desde un punto de vista moral, político y económico, afirmo que es urgente que el gobierno actúe con mayor eficacia en la ayuda al desarrollo de los paises »

2) Focusing .

a) restrictive

(51) « Estoy exclusivamente preguntando la hora. » (p. 431) 
Este complemento quedaría englobado en nuestra subclase 1 al modificar a una acción (preguntar). Esta construcción sería equivalente a la siguiente:

(51i) Estoy preguntando la hora sin otra intención

b) additive .

(52)《Juan está también llamando a María» (p. 433)

Lo mismo podría decirse de este complemento ya que modifica a un verbo de acción (llamar).

3) Intensifier .

a) emphasizer

(53)«Él realmente se sentó junto a ella. » (p.440)

En realidad, esta oración equivale a la siguiente construcción

(53i) Afirmo sinceramente que él se sentó junto a ella.

Estamos, pues, ante un complemento perteneciente a la subclase 1, ya que está modificando a un verbo de acción (afirmar)

b) amplifier.

(54)《 Ignoraron completamente mi petición. » (p. 445)

Estaríamos de nuevo ante un complemento perteneciente a la subclase 1, ya que está modificando a un verbo de acción (ignorar)

c) downtoner .

(55)《Ellos prácticamente le forzaron a dimitir » (p. 454)

Otro tanto sucede con este complemento.

4) Process.

a) Manner

(56) « Ella le habló con frialdad» (p. 460). 
Es un complemento perteneciente a la subclase 1, ya que modifica a un verbo de acción (hablar).

5) Subject .

(51) « Nos engañó deliberadamente » (p. 466)

Estamos ante un caso otra vez perteneciente a la subclase 1 con un verbo de acción (engañar).

6) Formulaic .

(58) «Le invitamos cordialmente a nuestra fiesta» (p. 470)

Este último caso de nuevo puede ser caracterizado como un complemento de modo perteneciente a la subclase 1 .

En conclusión, los esquemas sígnicos que caracterizarían a la clase de función 'complemento circunstancial' de modo serían los siguientes:

La subclase de modo 1 se definiría así:

\section{Subclase de modo 1}

Ausencia de concordancia con NP

Sintagmas preposicionales de modo, adverbios de modo, loc. adv. modo, prop. sub. adv. modo, construcciones gerundio y participo de modo con verbos de acción y de estado.

Periferia

Responsabilidad, control, volición 
La subclase de modo 2 se define así:

\section{Subclase de modo 2}

Ausencia de concordancia con NP

Sintagmas preposicionales de modo, adverbios de modo, locuciones adverbiales de modo, proposiciones subordinadas adverbiales de modo y contrucciones de gerundio y participio con verbos de experimentac.

\section{Periferia}

Modo

La subclase de modo 3 se define así:

\begin{tabular}{|c|}
\hline Subclase de modo 3 \\
\hline Ausencia de concordancia con NP \\
\hline $\begin{array}{l}\text { Prop. sub. adv. modo, adverbios y loc. adverbiales } \\
\text { de modo, sint. prep. de modo, construcciones } \\
\text { gerundio y participio con verb. estado y 'pseudo- } \\
\text { copulativos' }\end{array}$ \\
\hline Periferia \\
\hline Valoración/estimación \\
\hline
\end{tabular}

\section{BIBLIOGRAFÍA}

ACOSTA, L., «Las partículas modales en alemán y en español», Studia Philologica Salmanticiensia, 7-8, 1984, pp. 7-41.

ALARCOS, E., Estudios de gramática funcional del español, 2 edición, Madrid, Gredos, 1970/1973. 
-, Gramática estructural (según la Escuela de Copenhague y con especial atención a la lengua española), Madrid, Gredos, 1977.

--, « Metodología estructural y funcional en Linguiística », Revista Española de Lingüística, 7, 2, 1977.

—, «Prólogo» a H. Martínez García, El suplemento en español, Madrid, Gredos, 1986.

ALCINA FRANCH, J. y BLECUA, J. L., Gramática española, Barcelona, Ariel, 1975. ALLERTON, D. J.-GRUTTENDEN, A., «English sentence adverbials: Their syntax and their intonation in British English», Lingua, 34, 1974, pp.1-30.

BALLY, Ch., Linguistique générale et linguiistique française, Berna, Francke, 1965.

BARRENECHEA, A. M., «Operadores pragmáticos de actitud oracional: Ios adverbios en -mente y otros signos» AA.VV., Estudios lingüísticos y dialectológicostemas hispánicos, Argentina, Hachette, 1979, pp. 39-59.

BELLO, A., Gramática de la lengua castellana, Aula de Cultura de Tenerile, 1981.

BENEZECH, J. L., "Vers une approche de la sémiologie des adverbes démonstratifs de lieu en espagnol » Mélanges offerts a Ch. V. Aubrun, T. I, Paris, Éditions Hispaniques, 1973, pp.59-67.

BOSQUE, I., « Dos notas sobre el concepto de suplemento en la gramática funcional», Dicenda, 2, 1983, pp. 147-156.

—, Las categorías granaticales, Madrid, Síntesis, 1991.

CHAFE, W. L., Significado y estructura de la lengua., Barcelona, Planeta, 1976.

DANES, F., "The relation of Centre and Periphery as a Language Universal », Travaux Linguistiques de Prague, 2, 1966, pp.9-21.

DOMÍNGUEZ DE RODRÍGUEZ PASQUÉS, P., «Morfología y sintaxis del adverbio en -mente» en Actas del III Congreso Internacional de Hispanistas, México, 1970, pp. 293-303.

DYER, N. J., «A Study of the Old Spanish Adverb in -mente», Hispanic Review, 40, 1972, pp. 303-308.

EGEA, E. R., Los adverhios terminados en -mente en el español contemporáneo, Bogotá, Instituto Caro Cuervo, 1979.

FERREIRO-COUSO GONZÁLEZ, K., « Precedentes mediatos e inmediatos de los adverbios en -mente » Anales de filología hispánica, 5, 1990, pp. 281 1-300.

FUENTES, C., "El adverbio de frase«, RESLA, 3, 1987, pp. 55-74.

GILI GAYA, S., Curso superior de sintaxis española, 2" edición, Barcelona, Bibliograf, S.A, 1948/61/7.

GOLAY, J. P., «Le complément de manière est-il un complément de circonstance ?, Le français moderne, 32, 1, 1964, pp. 1-26.

GREENBAUM, S., Studies in English Adverbial Usage, London, Longman, 1969.

GREGORES, E., «Las formaciones adverbiales en -mente. Estudio descriptivo sobre el adverbio en español», Filología, 6, 1960, pp. 77-102.

GUIMIER, C., et P. LARCHER (ed.), L'adverbe dans tous ses états, Rennes, Travaiux linguistiques du CERLICO, 1991.

GUTIÉRREZ ORDÓÑEZ, S., «A propósito de Claúsulas y Oracione: », Archivum, XXI1-XXIII, 1977-78, pp. 529-547. 
—, «La determinación inmanente de las funciones en sintaxis», Contextos, II, 1983. pp.41-58.

—, « Sobre las categorías, las clases y la transposición », Contextos, 111/5. 1985, pp. 75-111.

HALLEBEEK, J., «El adverbio. Bosquejo de una posible morfosintaxis del elemento adverbial en español.» Dicenda, 1985, pp.35-56.

HEGER, K., «La conjugación objetiva en castellano y en francés», Teoriá Semántica 11, Madrid, Alcalál, 1974, pp. 87-106.

HERNÁNDEZ ALONSO, C., Gramática funcional del español, Madrid, Gredos, 1984. HERNANZ, M.LL., J. M. BRUCART, La sintaxis. I. Principios téricos. La oración simple, Barcelona, Crítica, 1987.

HUANG, S.-F., A Study of Adverbs. The Hague-Paris, Mouton, 1975.

JACKENDOFF, R., Semantic Interpretation in Generative Grammar, Cambridge (Mass.), MIT Press, 1972

- X Syntax. A Study of Phrase Structure, Cambridge (Mass.), MIT Press, 1977.

KNOWLES, D. R., « Análisis transformacional de una excepción en el sistema de los adverbios de modo » Actas del Cuarto Congreso Internacional de Hispanistas, vol. II, Salamanca, 1982. pp. 63-73.

KOKTOVA, E., Sentence Adverbials, Amsterdam, John Benjamins, 1986.

KOVACCI, O., «Sobre los adverbios oracionales» Homenaje a Ambrosio Rabanales, BFUCh, XXXI (1980-1981), pp. 519-535.

KURODA, S., «Some Remarks on English Manner Adverbials » Studies in General and Oriental Linguistics Presented to Shiro Hattori on the Ocasion of his Sixtienth Birthday, Tokyo, TEC Co. Ltd., 1970, pp. 375-396.

LAKOFF, G., Irregularity in Syntax, New York, Holt, Riverhart and Winston, Inc., 1970 .

- «Adverbios y operadores modales» en V. S. de Zavala, Semánica y sintaxis en la lingüistica transformatoria, 11, Madrid, Alianza Universidad, 1976.

-, « Linguistic Gestalts », Proceeding of Chicago Linguistic Society, 13, 1977.

LAPESA, R., «Sobre dos tipos de subordinación causal», Estudios ofrecidos a Emilio Alaros Llorach, 3, Oviedo, 1978.

LLORENTE, A., ; MONDÉJAR, J., «La conjugación objetiva en las lenguas románicas», RSEL, 4, 1, 1974, pp. 1-60.

LÓPEZ GARCÍA, A., «Problemas de clasificación semántica de los adverbios españoles en -mente» Actas XIV Congreso Internacional de Lingüistica y Filología Románica. Napoli, 1974, (1974), pp. 515-526.

- Elementos de semántica dinámica. Semántica española. Zaragoza, Pórtico, 1977.

- Estudios de lingüística española, Barcelona, Anagrama, 1983.

MARTINELLI, M., «Sur le distinction entre complements de verbe et de phrase »Linguisticae lnvestigationes, VIII, 1, 1984, pp. 195-199.

MARTÍNEZ GARCÍA, H., El suplemento en español, Madrid, Gredos, 1986.

NILSSON-EHLE, H., Les adverbes on $<<-$ ment $>>$ complements d'un verbe en fiansuis moderne (étude de classement syntaxique et sémantique), Lund, Eudes Romanes de Lund, 1941. 
POTTIER, B., «Problemas relativos a los adverbios en -mente» Lingüística moderna y filología hispánica, Madrid, Gredos, 1968.

QUIRK, R., GREENBAUM, S., LEECH, G. y SVARTVIK, A Grammar of Contemporary English, Londres, Longman, 1972

RAMÓN TRIVES, E., «En torno a los conceptos de transitividad, complementación y circunstancia desde la teoría actancial y causal : problemas hispánicos.» Anales Universidad de Murcia, XXV, 3-4, 1975, pp. 91-121.

-, Aspectos de semántica lingüístico-textual, Madrid, Alcalá/Istmo, 1979.

-, Estudios sintáctico-semánticos del español-I-. La dinámica interoracional, Murcia, Godoy, 1982.

—, « El estudio de la lengua en los tipos sintagmáticos latentes propiciados por los verbos locativos y los tipos semióticos pragma-lingüísticos oracionales o manifestativos ", Homenaje al Profesor Juan Barceló Jiménez, Murcia, Academia Alfonso X, 1990, pp. 547-575.

REAL ACADEMIA ESPAÑOLA, Gramática de la lengua española, Madrid, EspasaCalpe, 1917.

—, Esbozo de una nueva gramática de la lengua española,, Madrid, Espasa-Calpe, $1974 / 1989$.

ROCA PONS, J., Introducción a la gramática, Barcelona, Teide, 1972/1980.

ROJO, G., Cláusulas y oraciones, Univ. Santiago de Compostela, 1978.

—, «La función sintáctica como forma del significante», Verba, 6, 1979, pp. 107151

-, Aspectos básicos de sintaxis funcional, Málaga, Agora, 1983.

-, «En torno a los complementos circunstanciales» Lecciones del I y II curso de Lingüistica Funcional (1983-84), Universidad de Oviedo, 1985, pp.181-191.

SCHREIBER, P. A., «Some constraints on the formation of English Sentence Adverbs», Linguistic Inquiry, 2, 1, 1971, pp. 83-101.

—, « Style Disjuncts and the Performative Analysis », Linguistic Inquiry, 3, 3, 1972.

SROKA, K. A., The Syntax of English Phrasal Verbs, The Hague- Paris, Mouton, 1972.

TESNIÈRE. L., Eléments de syntaxe structurale, Paris, Klincksieck, 1976.

TRUJILLO, R, Elementos de semántica lingüística, Madrid, Cátedra, 1976.

VERA LUJÁN, A., «A propósito de las relaciones sintácticas oracionales: categorías y clases de funciones», Estudios de Lingüistica, 5, 1988-1989, pp.127-144. —,Las construcciones pronominales pasivas e impersonales en español, Murcia, Universidad de Murcia, 1990.

VERA LUJÁN, A. - Mª L. MASIÁ CANUTO, «La categoría de función ‘complemento circunstancial' en español», Voz y letra, II, 1, 1991, pp. 51-75.

ZIERER, E., «Algunos casos particulares de expresiones adverbiales y modales en los idiomas alemán y español» Beiträge zur Romanischen Philologie, XIV/1975 Heft 2, pp. 315-317 